\title{
Comercio tradicional de productos de gran consumo en Colombia: Movilidad del consumidor
}

\section{The traditional packaged consumer goodstradein Colombia: consumer mobility}

\author{
Emperatriz Londoño-Aldana1*, María E. Navas-Ríos \\ ${ }^{1}$ Economista, PhD. ${ }^{2}$ Administradora de Empresas, $\mathrm{PhD}$ \\ *Docentes Universidad de Cartagena - Colombia
}

Recibido: Marzo 31 de 2010. Aprobado: Enero 28 de 2011

\section{RESUMEN}

El crecimiento acelerado de las grandes superficies tanto nacionales como extranjeras en Colombia a partir de mediados de la década del noventa y acorde con las teorías que intentan explicar la evolución del comercio minorista hizo suponer que los pequeños comerciantes de productos de gran consumo se extinguirían en el corto tiempo.

El dinamismo del comercio, adaptándose a la evolución de la sociedad, como afirma Rebollo (1993), convierte rápidamente en obsoletas las clasificaciones al recoger características o funciones que ya no son útiles, sin embargo en el país el fenómeno, en lo referente a las tiendas de barrio, lejos de perder vigencia, se han convertido, en conjunto, en el principal competidor de las grandes cadenas de supermercados.

Palabras clave: Tienda de barrio, minorista, comercio tradicional, venta al detalle.

\begin{abstract}
The rapid growth of large-scale domestic and foreign sales coverage in many parts of Colombia since the mid-1990s, in line with theories explaining retail trade evolution, presumed that the small-scale consumer packaged goods traders would become extinct within a short time.

The dynamism of trade adapting to society's evolution, as stated by Rebollo (1993), has quickly made obsolete those classifications grouping features or functions which are no longer use ful. However, the social phenomenon in Colombia regarding corner shops, shows that far from becoming obsolete, they have become, collectively, the main competitor for large supermarket chains.
\end{abstract}

Key Words: grocery, retail, traditional trade, retail. 


\section{INTRODUCCIÓN}

El proceso de crecimiento y adaptación de las tiendas de barrio, se encuentra estrechamente relacionado con la situación económica del país y la consecuente reducción del ingreso familiar y aumento de los índices de pobreza; a nivel de compras de productos de consumo masivo el resultado ha sido una gradual sustitución del supermercado por la tienda en unos casos y en otros, ésta se ha convertido en el único canal al que gran parte de la población puede acceder; a esto se suman por una parte, el arraigo cultural con la tienda y por otra, la focalización de la industria en el pequeño comerciante, al "descubrir" su potencial total de distribución y su margen de cobertura del mercado.

La evolución del comercio al detalle ha sido explicada por diferentes teóricos y académicos lo que ha conducido a la formulación de teorías y modelos de la venta al detalle. La presente investigación se fundamenta en la rueda en espiral (Agergaard et al., 1970), que integra las teorías cíclicas, del conflicto y las medioambientales por una parte, y por la otra el principio de la polaridad de la venta al detalle (Brown, 1987, Dressman y Schary, 1960 y Kirby, 1976), toda vez que el comercio minorista de productos de gran consumo en Colombia se encuentra polarizado entre las tiendas de barrio y las grandes superficies.

El modelo CREM (Evolución del modelo de combinación minorista), o Rueda en Espiral, se basa en las tres teorías de evolución de venta al por menor más comúnmente usadas: la teoría cíclica (ciclo de precio: mínimo - alto - bajo (McNair, 1958); ciclo de surtido: general -específico - general (Hollander, 1966), la teoría de conflicto (síntesis empresa inicial)antítesis (empresa competidora) - tesis (nueva empresa con características de las dos anteriores (Gist, 1968; Oren, 1989) y la teoría del entorno o medioambiental (variables del entorno, afectan la evolución de las instituciones detallistas: demográficas, socioeconómicas, legales, geográficas de competencia y tecnológicas (Brown, 1987; Gist, 1968; Oren, 1989).

Los tres principios teóricos en los que se fundamenta la Rueda en espiral son:

a. Estructuras rítmicas del cambio en espiral (figura 1. primera parte del esquema)

b. Efectos del conflicto o cambios en la competencia (figura 1. primera parte del esquema)

c. Influencia del ambientes de venta al por menor (figura 1. segunda parte del esquema)

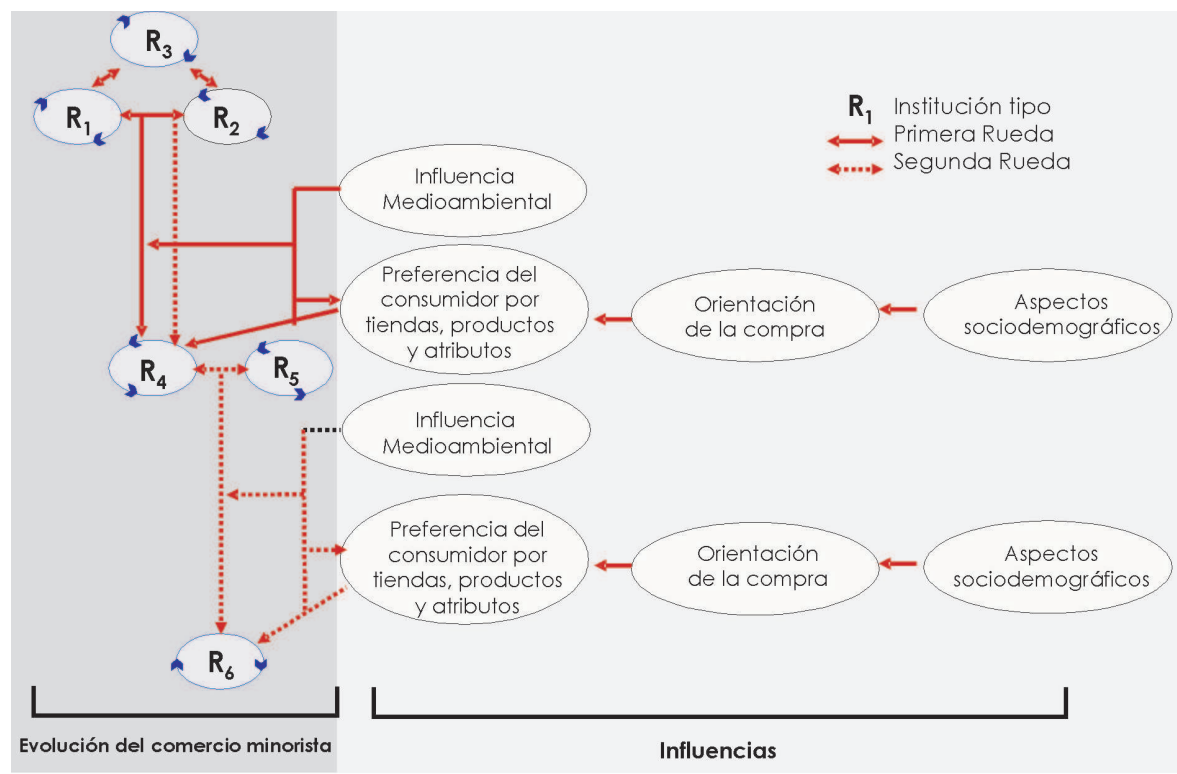

Fuente: Adaptado de Agergaard, Olsen y Allpass(1970) The interaction between retailing and the urban center structure: A theory of spiral movement. Environment and planning, 2, 55-71

Figura 1. Rueda en espiral 
Al combinar la teoría cíclica con la del conflicto, los autores muestran que el proceso de evolución de las instituciones detallistas tipo, presentan cambios cíclicos en espiral en la secuencia $R_{1}$ a $R_{6}$.

Con el movimiento en espiral (Principio de las estructuras rítmicas de cambio en espiral), la institución tipo " $R_{1}$ " gira en sentido contrario a la institución tipo " $\mathrm{R}_{2}$ ", debido a que se encuentran en conflicto; surge entonces, una nueva institución " $\mathrm{R}_{4}$ ", como resultado del conflicto o de la fricción de la competencia. El modelo de Agergaard, et al (1970), igualmente permite observar el conflicto entre más de dos instituciones tipo (" $R_{1}$,", $R_{2}$ ", " $R_{3}$ ").

En el tiempo, la nueva institución tipo " $\mathrm{R}_{4}$ ", empieza su evolución en espiral y regresa a un nivel más alto que el que tenía cuando la rueda empezó a moverse. La repetición del proceso continúa en la medida en que igualmente se repite el conflicto, con el consecuente surgimiento de nuevas instituciones tipo: " $R_{4}$ " entra en conflicto con, $R_{5}$ " y surge," $R_{6}$ ", etc.

Al mirar el modelo de la Rueda en espiral, desde la teoría del entorno, se observa que durante el proceso de creación de una nueva institución minorista tipo ("R $R_{4}$ "), distintos factores (sociales, culturales, económicos, geográficos, técnicos y legales) ejercen influencia sobre ella. Por otra parte, las preferencias de los consumidores por los atributos de la tienda o por los de los productos cambian; estas preferencias a su vez, están influenciados por la orientación de las comprar y

\section{MATERIALES Y MÉTODOS}

Para efectos de la captura de datos, se trabajó en dos sentidos: uno, observación mediante la aplicación de un formulario estructurado y otro, la aplicación de una encuesta estructurada con objetivo encubierto, en la que para establecer opinión, actitud o percepción del encuestado se utiliza una escala de Likert de 5 puntos para valorar ésta por los factores sociodemográficos (Monroe y Guiltinan, 1975; Sheth 1983; Shim y Kotsiopulos 1992).

Agergaard et al. (1970), separan al consumidor del grupo de factores medioambientales; esto se debe a que ellos consideran al consumidor como el mayor influenciador e interactuante en la venta al por menor. Algunos investigadores como McNair (1958) y Gist (1968) no señalan el papel del consumidor en el proceso de cambio de la venta minorista, otros como Blizzard (1976) ni siquiera Io mencionan Kim (2003).Igualmente investigadores como Monroe y Guiltinan (1975) y Arnold et al (1998), si presentan la capacidad del consumidor para actuar directamente y ejercer influencia en la evolución de la venta al por menor.

De acuerdo con MacNair y May (1978) y Sheth (1983), las necesidades de un consumidor por un cierto tipo de tienda minorista están influenciadas por aspectos económicos, tecnológicos y la estructura social. En razón a esta explicación, la preferencia de los consumidores por los atributos de la tienda y/o los productos y las influencias ambientales asociadas son propuestas en la Rueda en Espiral para tener una relación causa efecto.

El presente trabajo se deriva de la línea de investigación sobre comercio minorista y se mira desde la perspectiva de las influencias sobre el consumidorcomo se presenta en el modelo CREM o Rueda en Espiral.

grados de acuerdo/desacuerdo. Esta escala ha sido validada con el coeficiente Alpha de Cronbach. En el procesamiento de los datos se utilizo el software Dyane (Análisis y diseño de Encuestas) cuya versatilidad, además de las estadísticas básicas permite hacer otros análisis necesarios como el factorial de correspondencias, clusters $y$ redes neuronales artificiales. 


\section{RESULTADOS Y DISCUSIÓN}

\section{CREM - ASPECTOS SOCIODEMOGRAFICOS Y SOCIECONOMICOS}

La crisis económica afrontada por el país en 1999 y su lenta recuperación, con su consecuente contracción de los ingresos familiares y el achatamiento de la pirámide social, unidos a la cultura de la tienda, han favorecido el incremento tanto de las tiendas como de la demanda por este tipo de establecimientos, produciéndose las siguientes situaciones:

- Migración de compradores en supermercado hacia la tienda de barrio.

- Aumento del comprador que depende del crédito en la tienda.

- La tienda como alternativa para la generación inmediata de ingresos en los hogares.

La pirámide social propuesta por Alameda (2003) en la figura 2, habla por sí sola, en términos de la desproporción de la distribución de los ingresos frente a la distribución de la población, se observa que mientras que el $53 \%$ de la población vive con menos de 192 dólares al mes, el $0.01 \%$ lo hace con más de 52.000. Igualmente dentro del rango de ingresos que podrían considerarse medios (US\$371 y US\$2.400), solamente se encuentra el $12 \%$.

Esta situación ha llevado a los consumidores a replantear tanto la cantidad como la calidad de los productos de su respectiva canasta familiar y de igual manera su proveedor. Dicho replanteamiento hace referencia a la disminución de las cantidades usualmente adquiridas buscando productos en presentaciones más pequeñas y/o fraccionamiento de los mismos y la sustitución de unas marcas por otras de menor valor.

\section{DISTRIBUCIÓN DE LA POBLACIÓN INGRESOS FAMILIARES DOLARES AL MES}

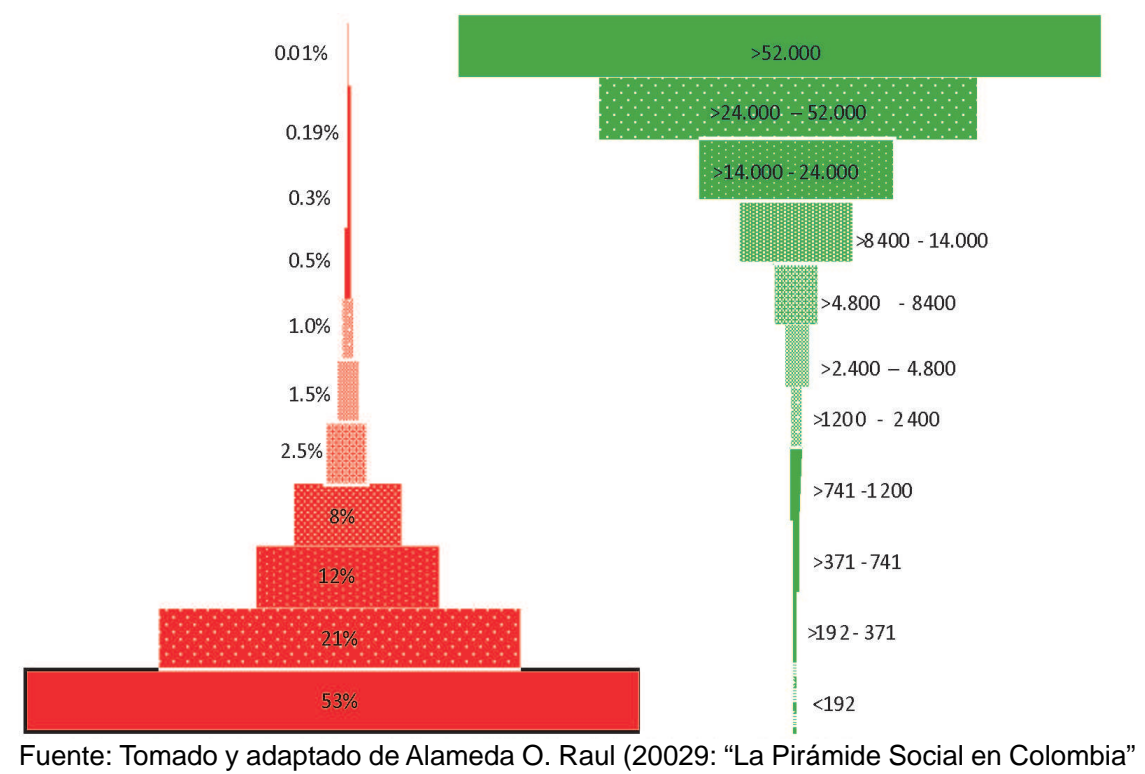

Figura 2. Pirámide Social

En la Tabla 1, se observa la estratificación oficial de seis niveles o estratos ( 6 y 5 alto, 4 y 3 medio 2 y 1 bajo) propuesta por Alameda (2003), lo cual no refleja la real estructura social del país; por ello los indicadores presentados por este autor, referidos a niveles de ingreso y necesidades básicas insatisfechas, se acercan más a la realidad actual: el $95 \%$ de la población en Colombia es pobre. 
El $53 \%$ de la población se encuentra por debajo de la línea de pobreza, por tanto, la única opción posible para abastecerse es la tienda de barrio, donde además del crédito puede con un bajo desembolso adquirir lo necesario para una alimentación muy básica. (No se trata de bajo precio, sino de la variedad de productos que se alcanza a comprar con un bajo desembolso, toda vez que el tendero fracciona o menudea los productos).

Existe un $41 \%$ considerado pobres, a su vez dividido en tres categorías: muy pobre $(21 \%)$, medianamente pobre (12\%) y menos pobre $8 \%$.; este grupo integra la masa crítica disputada por supermercados y tiendas de barrio.

Tabla 1. Colombia: Estratificación socioeconómica

\begin{tabular}{llllr}
\hline & \multicolumn{1}{c}{ Estrato } & & Subestrato & \% Población \\
\hline 1 & Marginal o miserable & & & 53.0 \\
& SUBTOTAL MARGINAL & & 53.0 \\
2 & Pobre & 2 & Muy pobre & 21.0 \\
3 & & 3 & Medianamente pobre & 12.0 \\
4 & & 4 & Menos pobre & 8.0 \\
& SUBTOTAL POBRES & & & 41.0 \\
5 & Acomodado & 5 & Menos acomodado & 2.5 \\
6 & & 6 & Medianamente acomodado & 1.5 \\
7 & & 7 & Más acomodado & 1.0 \\
& SUBTOTALACOMODADOS & & 5.0 \\
8 & Rico & 8 & Menos rico & 0.5 \\
9 & & 9 & Medianamente rico & 0.3 \\
10 & & 10 & Más rico & 0.199 \\
& SUBTOTAL RICOS & & & 0.999 \\
11 & Potentado & & & 0.001 \\
& SUBTOTAL POTENTADOS & & 0.001 \\
& TOTAL & & 100.0 \\
\hline
\end{tabular}

\section{CREM - ORIENTACION DE LA COMPRA}

El mercado de productos de gran consumo, de acuerdo con la concentración de los puntos de venta al detalle, se encuentra distribuido así: el $74 \%$ almacenes de los grandes minoristas se focaliza en los estratos socioeconómicos medios y altos de la población y en los estratos medios bajos y bajos se encuentra el $94 \%$ de las tiendas de barrio.

Como se anotó anteriormente el 95\% de la población es pobre y más de la mitad vive por debajo de la línea de pobreza; en estas condiciones el mercado de productos de gran consumo jalona hacia el grueso de la población que se ubica en los estratos socioeconómicos medios y bajos.

Solamente el $0,5 \%$ compra sólo en supermercados, el 5,5\% lo hace mayoritariamente en supermercados e hipermercados, seguido de superetes; el $20 \%$ distribuye sus compras entre tiendas de barrio, plazas de mercado, superetes y supermercados; el $21 \%$ compra mayoritariamente en tiendas de barrio y el $53 \%$ únicamente se puede proveerse en estos establecimientos. figura 3.

\section{CREM - PREFERENCIA DEL CONSUMIDOR POR LA TIENDA DE BARRIO}

Los motivos por los cuales las personas que han comprado o compran en la tienda de barrio, se encuentran asociados con diversos aspectos de orden económico, comercial y cultural. Cada grupo de variables presenta un peso diferente en la decisión de compra del consumidor; en su orden se encuentran: comerciales, socioculturales y económicas. 


\begin{tabular}{|c|c|c|c|}
\hline $\begin{aligned} \text { Ingreso/mes } & \\
& <52.000 \\
24.000 & <52.000 \\
14.000 & <24.000\end{aligned}$ & $0.5 \%$ & $\mid$ & Sólo en supermercados \\
\hline $\begin{array}{l}8.400<14.000 \\
4.800<8.400 \\
2.400<4.800 \\
1.200<2.400\end{array}$ & $5.5 \%$ & $\begin{array}{c}0.5 \% \\
1.0 \% \\
1.5 \% \\
2.5 \% \\
\end{array}$ & $\begin{array}{l}\text { Mayoritariamente en supermercados e } \\
\text { hipermercados, seguido de superetes }\end{array}$ \\
\hline $\begin{array}{r}\quad 741<1.200 \\
371<741\end{array}$ & $20 \%$ & $\frac{8 \%}{12 \%}$ & $\begin{array}{l}\text { Proporcionalmente en tiendas de barrio, } \\
\text { plaza de mercado, superetes } \\
\text { y supermercados }\end{array}$ \\
\hline $192<371$ & & $21 \%$ & Mayoritaria en tiendas de barrio \\
\hline$<192$ & & $53 \%$ & Sólo en tiendas de barrio \\
\hline
\end{tabular}

Fuente: Tomado y adaptado de Alameda O. Raul (20029: "La Pirámide Social en Colombia"

Figura 3. Dónde compran los consumidores en Colombia?

Tradicionalmente en Colombia las personas han comprado en la tienda de barrio, bien sea para proveerse de todos los productos de la canasta familiar o para reponer los faltantes de despensa. Este comportamiento luego de la crisis económica vivida por el país a finales de los noventas y comienzos de los dos mil, llevó a la contracción de la demanda por productos de gran consumo comprados en los supermercados e incremento de la misma por la tienda de barrio; es decir, se produjo una migración de compradores del supermercado a la tienda de barrio.

La aplicación de una encuesta a 191 personas (1 por hogar) ha permitido conocer cuáles son los motivos que han llevado a las familias a continuar comprando en la tienda y/o regresar a ella e incrementar los montos adquiridos.

El análisis de once motivos iniciales, en una pregunta multirrespuesta, permitió la escogencia de seis, tomando como base una frecuencia mínima de $73 \%$. Por aparte la tabulación individual de la opinión acerca de dichos motivos mostró que la frecuencia de calificación mediana y totalmente de acuerdo, igualmente se encuentra por encima de $73 \%$. Los resultados ordenados de mayor a menor se presentan en la tabla 2.

Tabla 2. Motivos de traslado y/o aumento de compras en la tienda de barrio

\begin{tabular}{llcc}
\hline MOTIVOS & $\begin{array}{c}\text { \% de } \\
\text { Respuesta } \\
\text { Conjunta }\end{array}$ & $\begin{array}{c}\text { \% Respuesta } \\
\text { individual\% }\end{array}$ \\
\hline 1 & Trata amistoso & 100 & 90.9 \\
\hline 2 & Cercanía a la tienda & 91 & 100 \\
\hline 3 & No rigurosidad en presentación & 91 & 72.9 \\
\hline 4 & Fraccionamiento a pedidos & 82 & 90.9 \\
\hline 5 & Tamaño de la presentación & 73 & 90.9 \\
\hline 6 & Número de tiendas cercanas & 73 & 100 \\
\hline
\end{tabular}


Al analizar estos seis primeros motivos, según la categoría de variables establecida, se presenta una primacía los motivos comerciales, seguidos de los culturales y finalmente los económicos; ellos aunque no figuran dentro de los seis primeros, si son tenidos en cuenta dentro de los diez principales motivos que han llevado al consumidor a incrementar la compra en las tiendas de barrio. Luego de la depresión anotada, se produjo una fuerte y efectiva respuesta de los comerciantes para adaptarse a las nuevas necesidades de los consumidores y se dio un vuelco total a la forma como se había venido dando la comercialización y distribución de los productos de consumo masivo. El resultado ha sido la generación de unos consumidores conscientes de una realidad (disminución de sus ingresos), quienes tienen la oportunidad de acceder a unos beneficios comerciales actuales los cuales se han convertido en definidores de su traslado del supermercado a la tienda de barrio.

Un $20 \%$ de los consumidores llevan 10 y más años comprando en la tienda, mientras que el $80 \%$ lo hace desde hace cinco años; esto se corrobora con la presencia de solamente un $18,26 \%$ no ha incrementado sus compras en la tienda y disminuido en el supermercado.

Un comportamiento similar se encuentra en la calificación dada a las siguientes afirmaciones: $\mathrm{Si}$ en el supermercado encontrara los productos en las presentaciones que ofrecen en las tiendas

\section{CONCLUSIONES}

En Colombia las tiendas de barrio conservan su protagonismo dentro del canal de distribución de productos de gran consumo, tanto por el incremento en la demanda por este tipo de establecimientos. Estos factores las han llevado, por una parte a tener más del $60 \%$ de participación en el mercado y por otra, a captar el interés por parte de los industriales quienes son conscientes de su potencial de compra y buscan proveerlos de los productos a la medida de las necesidades de sus clientes. aumentaría el monto de mis compras en ese sitio; y He vuelto a comprar en la tienda porque no encuentro diferencia significativa en la relación cantidad precio de los productos que en ella encuentro frente a los que me ofrece el supermercado; los que siempre han comprado en tiendas se encuentran en total desacuerdo con estas afirmaciones (18.18\% y $27.27 \%$ respectivamente), el restante porcentaje expresa estar en total y medianamente acuerdo, se mantienen en la misma proporción.

El orden prioritario de los motivos de la migración de los supermercados a las tiendas de barrio se demuestra también mediante el análisis de los valores medios ordenados de mayor a menor. De un total de 13 motivos, tomados sobre la base de una calificación superior a 3,5, siete pertenecen a la categoría comercial, cuatro a la categoría cultural y dos a la económica (tabla 2).

A nivel comercial son mejor calificados los factores referentes al número de tiendas cercanas a la vivienda $(4,5)$, su ubicación $(4,4)$ y la devolución de productos $(4,03)$. Tabla 3 A.

En los culturales se privilegia la amplitud del horario ya que el comerciante abre en promedio a las 6 a.m y cierra alrededor de las 10 de la noche. Dentro de los motivos económicos, los encuestados resaltan el tipo de crédito que le otorga el tendero $(3,8)$, los costos de tiempo y transporte para ir al supermercado $(3,5)$. Tabla 3.

El pequeño comerciante (tienda de barrio), cuenta con una flexibilidad en el manejo de su clientela, productos (tamaño y fraccionamiento), precios, crédito y atención personalizada que no puede ofrecer el supermercado o el hipermercado. Esto unido a las condiciones económicas de la población y al arraigo de la tienda en los hogares colombianos, permite vislumbrar la consolidación de tales establecimientos en el mercado de productos de gran consumo. 
Tabla 3. Motivos de traslado del supermercado a la tienda de barrio

(Tabulación de valores medios)

\begin{tabular}{|c|c|c|c|}
\hline Var & Afirmación & $\begin{array}{c}\text { Media } \\
\text { aritmética }\end{array}$ & $\begin{array}{c}\text { Desviación } \\
\text { estandar }\end{array}$ \\
\hline \multicolumn{4}{|c|}{ A. Motivos comerciales } \\
\hline 15 & $\begin{array}{l}\text { Cerca de mi vivienda dispongo de tres o más tiendas para } \\
\text { hacer mis compras }\end{array}$ & 4,4555 & 0,823 \\
\hline 17 & $\begin{array}{l}\text { Considero que una de las grandes ventajas que ofrece la } \\
\text { tienda es su ubicación }\end{array}$ & 4,4398 & 0,6673 \\
\hline 25 & $\begin{array}{l}\text { Las devoluciones que aceptan en la tienda no las aceptan en } \\
\text { los supermercados }\end{array}$ & 4,0366 & 0,9728 \\
\hline 8 & $\begin{array}{l}\text { En el supermercado compro principalmente los productos } \\
\text { que me significan una buena promoción }\end{array}$ & 3,8796 & 1,1537 \\
\hline 24 & $\begin{array}{l}\text { En el supermercado recibo la amabilidad del empleado para } \\
\text { cualquier cliente }\end{array}$ & 3,7539 & 1,1149 \\
\hline 5 & $\begin{array}{l}\text { Compro en la tienda porque me adaptan las cantidades y } \\
\text { precios del producto que necesito al dinero disponible en el } \\
\text { momento. }\end{array}$ & 3,6754 & 1,0923 \\
\hline 16 & $\begin{array}{l}\text { Me gustan las condiciones higiénicas de la tienda donde hago } \\
\text { mis compras }\end{array}$ & 3,644 & 1,1929 \\
\hline \multicolumn{4}{|c|}{ B. Motivos socioculturales } \\
\hline 12 & $\begin{array}{l}\text { La tienda es un lugar al que puedo acceder a tempranas } \\
\text { horas del dia o tarde de la noche (amplitud de horario). } \\
\text { Prefiero comprar en la tienda porque está muy cerca de mi }\end{array}$ & 4,4555 & 0,7567 \\
\hline 14 & vivienda. & 3,8429 & 1,2766 \\
\hline 22 & $\begin{array}{l}\text { En la tienda recibo el trato personalizado y amistoso que no } \\
\text { me dan en el supermercado } \\
\text { Me gusta ir a comprar a la tienda porque no me exige }\end{array}$ & 3,6335 & 1,2115 \\
\hline 23 & $\begin{array}{l}\text { Me gusta ir a comprar a la tienda porque no me exige } \\
\text { rigurosidad en cuanto a la presentación personal. }\end{array}$ & 3,5916 & 1,0389 \\
\hline \multicolumn{4}{|c|}{ C. Motivos económicos } \\
\hline 18 & $\begin{array}{l}\text { Los costos de tiempo y transporte para ir al supermercado, m } \\
\text { e llevan a preferir comprar en la tienda } \\
\text { El tipo de crédito que me otorga el tendero no me lo ofrece el }\end{array}$ & 3,8168 & 1,168 \\
\hline 19 & supermercado & 3,5602 & 1,2555 \\
\hline
\end{tabular}

\section{REFERENCIAS}

Agergaard E, Olsen PA, Allpass J. The interaction between retailing and the urban center structure: $A$ theory of spiral movement. Environment and Planning 1970; 2: 55-71.

Kim SH, Kincade DH. The Model for the Evolution of Retail Institution Types in South Korea. JTATM: Journal of Textiles and Apparel. Winter 2006; 5(1):1-29.

Alameda OR. La Pirámide Social en Colombia. Revista Nueva Colombia 2003; 1:18-23.
Arnold S J, Handelman J, Tigert DJ. The impact of a market spoiler on consumer preference structures (or, what happens when Wal-Mart comes to town). Journal of Retailing and Consumer Services 1998;5(1): 1-13.

Blizzard RT. (1976): The Competitive Evolution of Selected Retail Institutions in The United States and Australia: a Culture Ecological Analysis. Disertación Tesis Doctoral, University of Colorado. 
Brown S. An Integrated Approach to Retail Change: The Multi-Polarisation Model. The Service Industries Journal 1987; 7 (2):153-164.

Crombach LJ. (1951) Coeficient Alpha and the Internal Structure of Test, Psychometrika. En: antesmases Miguel (2005) 297-334pp. DYANE (Versión 3). Diseño y Análisis de Encuestas en Investigación Social y de Mercados, Editorial Pirámide, Madrid.

Dreesmann ACR. Patterns of evolution in retailing. Journal of Retailing 1968; 44:64-81.

Guiltinan JP. Planned and Evolutionary Changes in Distribution Channels. Journal of Retailing 1974; 50(2):79-91.

Gist RR (1968). Retailing: Concepts and decisions. New York: John Wiley and Sons. 109 - 110pp.

Hollander SC. The Wheel of Retailing. Journal of Marketing 1960; 24:37-42.

Hollander SC (1970): Multinational Retailing. Institute for International Business and Economic Development Studies, Michigan State University (East Lansing).

Hollander SC. Retailers as Creatures and Creators of the Social Order. International Journal of Retail \& Distribution Management 2002; 30 (11):514-517.

Hollander SC. Notes on the Retailing Accordion. Journal of Marketing 1966; 42: 29-40.

Cuesta VP (2001). Estrategias de Crecimiento de las Empresas de Distribución Comercial de Productos de Gran Consumo que Operan en España". Tesis Doctoral, Universidad Autónoma de Madrid - España.

Kim Sook-Hyun (2003): The Model for the Evolution of Retail Institution types in South Korea. Tesis
Doctoral the Parment of Apparel Housin and Research Management Clothing \& Textiles Program.

Virginia tech Blacksburg, VA. Faculty of the Virginian Polytechnic Institute State University.

Kirby DA (1976): The North American Convenience Store: Implications for Britain, In Jones, $P$, Oliphant R .(Eds.), Local Shops: Problems and Prospects, Reading, Unit for Retail Planning Information, 1976, Pp 95-100. En Brown S. Institutional Change in Retailing. A Review and Synthesis. European Journal of Marketing. 1987; 21 (6):5-36.

Mcnair MP (1958): "Significant Trends and Developments In the Post War Periods", En Smith, A. B.(Ed.), Competitive Distribu-Tion in a Free High Level Economy and Its Impact for the University, University of Pittsburgh Press, Pittsburgh, Pp. 125, 125. Cuesta Valiño, Pedro (2001), Estrategias de Crecimiento de las Empresas de Distribución Comercial de Productos de gran Consumo que Operan en España, Tesis Doctoral, Universidad Autónoma de Madrid, Madrid.

Mcnair MP, May EG. The Next Revolution of the Retailing Wheel. Harvard Business Review 1978; 56(5):81-91.

Oren C. The Dialectic of the Retail Evolution. Journal of Direct Marketing 1989; 3 (1): 15-29.

Rebollo A. Clasificación de las formas comerciales: el producto-establecimiento. Distribución y Consumo. 1993; 3 (10): 10-18.

Santesmases M. (2005): DYANE (Versión 3). Diseño y Análisis de Encuestas en Investigación Social y de Mercados, Editorial Pirámide, Madrid.

Sheth J. (1983). An Integrative Theory of Patronage Preference and Behavior. In W. Darden \& R. Lusch (Eds.), Patronage Behavior and Retail Management. New York: North Holland. 